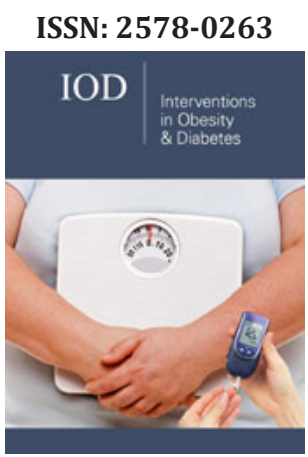

*Corresponding author: Prof. Diego Ferone, Endocrinology Unit, Department of Internal Medicine \& Medical Specialties (DIMI), Center of Excellence for Biomedical Research (CEBR), University of Genova, Italy. Email: ferone@unige.it

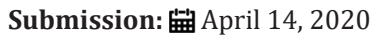

Published: 㸃 May 14, 2020

Volume 4 - Issue 1

How to cite this article: Eleonora Monti, Alberto Rebora, Diego Ferone, Enrico Torre. Treatment of Type 2 Diabetes in Subjects with Obesity: What is the Best Approach?. Interventions Obes Diabetes 4(1). IOD.000579. 2020. DOI: 10.31031/IOD.2020.04.000579

Copyright@ Diego Ferone. This article is distributed under the terms of the Creative Commons Attribution 4.0 International License, which permits unrestricted use and redistribution provided that the original author and source are credited.

\section{Treatment of Type 2 Diabetes in Subjects with Obesity: What is the Best Approach?}

\author{
Eleonora Monti ${ }^{1}$, Alberto Rebora ${ }^{1}$, Manuela Albertelli ${ }^{2,3}$, Claudia Campana ${ }^{2,3}$, \\ Federico Gatto ${ }^{2,3}$, Diego Ferone ${ }^{2,3 *}$ and Enrico Torre ${ }^{1}$ \\ ${ }^{1}$ Endocrinology, Diabetology and Metabolic Diseases Unit, ALS3, Genova, Italy \\ ${ }^{2}$ Endocrinology, Department of internal Medicine \& Medical Specialties (DIMI) and Center of Excellence \\ for Biomedical Research (CEBR), University of Genova, Italy \\ ${ }^{3}$ Endocrinology, IRCCS Ospedale Policlinico San Martino, Genova, Italy
}

\begin{abstract}
Prevalence of both obesity and type 2 diabetes (T2DM) is increasing worldwide. Obesity, along with insulin resistance, predisposes individuals to low-grade chronic inflammation. Moreover, the combination of insulin resistance and hyperinsulinemia gives rise to the metabolic syndrome. The management of obesity can delay the progression to diabetes and first line treatment is represented by interventions on lifestyle (low-calorie diet and aerobic exercise). In obese patients with T2DM, weight loss improves glycaemic control and, therefore, reduces the antidiabetic drug need. While some older medications, including insulin, result in weight gain, the new molecules (glucagon-like peptide receptors agonists [GLP-1ra] and sodium-glucose co-transporter 2 inhibitors [SGLT2i]) result in weight loss. GLP-1ra has an anorexic action because it slows emptying gastric, whereas SGLT2i induce glycosuria by an osmotic diuresis associated with a loss of water. To date, metformin is used as a first-line anti-diabetic drug. This molecule is known to reduce hepatic gluconeogenesis, to decrease intestinal absorption of glucose and to improve peripheral glucose uptake. In obese patients with insulin resistance metformin can correct this alteration and promote weight loss.
\end{abstract}

In conclusion, significant interventions on patient's lifestyle are crucial to treat both diabetes and obesity. These can be seen as the two faces of the same disease, the metabolic syndrome. Metformin is the first-line therapy in T2DM for its tolerability and efficacy in reducing glycated hemoglobin. Thanks to their different mechanism of action, metformin in association with GLP-1ra and/or SGLT2i probably represent the best choice for obese patients with T2DM.

Keywords: Diabetes mellitus, Obesity, Metabolic syndrome, Antidiabetic drugs

Abbreviations: T2DM: Type 2 Diabetes Mellitus; BMI: Body Mass Index; NEFA: Nonesterified Fatty Acids; GLP-1ra: Glucagon-Like Peptide Receptors Agonists; SGLT2i: Sodium-Glucose Co-Transporter 2 Inhibitors; DPP-IVi: Dipeptidyl Peptidase-4 Inhibitors; FDA: Food and Drug Administration; EMA: European Medicines Agency; ADA: American Diabetes Association

\section{Introduction}

The correlation between type 2 diabetes (T2DM) and obesity was remarkable by Sims and colleagues in the 1970s when they coined the term "diabesity" [1]. The prevalence of both T2DM and obesity has continuously increased worldwide. It has been predicted that in 2030 the prevalence of T2DM will reach more than $7.0 \%$ of the world's population, paralleling the body mass index (BMI). The precise pathways leading to T2DM are not yet completely known, however, the current understanding of its pathophysiology recognizes, in most cases, obesity and physical inactivity as start-up processes [2]. The increased adipose tissue mass, mostly at visceral site, leads to elevated plasma nonesterified fatty acids (NEFA) concentrations, and these in turn leads to insulin resistance in insulin target tissues, such as muscle and liver [3]. The insulin resistance state represents a major stress on the pancreatic $\beta$-cells to increase their insulin secretion in order to offset the blunted insulin action. As long as the $\beta$-cells are able to increase their secretion of insulin sufficiently to overcome the insulin resistance, glucose tolerance is kept at normal levels. However, over time the $\beta$-cells begin to fail and initially the postprandial plasma glucose levels and subsequently the fasting plasma glucose 
concentration begin to rise, leading to the onset of overt type 2 diabetes [4]. Obesity, along with insulin resistance, predisposes individuals to low-grade chronic inflammation and metabolic complications. Further, the combination of insulin resistance and subsequent hyperinsulinaemia gives rise to a number of metabolic and cardiovascular changes that bring to metabolic syndrome, typically characterised by T2DM, obesity, dyslipidaemia, coronary artery disease and hypertension [5,6]. This picture is summarized in (Table 1). Moreover, obesity represents the major determinant of musculoskeletal and osteoarticular weight related diseases [7].

Table 1: Criteria of metabolic syndrome.

\begin{tabular}{|r|}
\hline Waist circumference $>94 \mathrm{~cm}$ in men and $>80 \mathrm{~cm}$ in women \\
\hline Triglycerides levels $\geq 1.7 \mathrm{mmol} / \mathrm{L}$ or specific treatment for this lipid abnormality \\
\hline HDL cholesterol $<1.03 \mathrm{mmol} / \mathrm{L}$ in males and $<1.29 \mathrm{mmol} / \mathrm{L}$ in females \\
\hline Systolic blood pressure: systolic $\geq 130$ or diastolic $\geq 85 \mathrm{mmHg}$ or treatment of previously diagnosed hypertension \\
\hline Glucose levels $\geq 5.6 \mathrm{mmol} / \mathrm{L}$ or previously diagnosed type 2 diabetes \\
\hline
\end{tabular}

\section{Therapy}

Obesity management can delay the progression of glucose intolerance to T2DM [8]. First line therapy for obesity is represented by lifestyle interventions such as low-calorie diet and aerobic exercise in order to reduce insulin resistance and increase GLUT-4 expression by skeletal muscles. About 300 minutes/week of endurance activity at moderate intensity, or even 150 minutes of more intense activity, can mobilize visceral fat that is correlated with the high cardiovascular risk. The general prescription for the population is at least of 150 minutes/week of moderate aerobic activity, equivalent to walking at $5-6 \mathrm{~km} / \mathrm{h}[9,10]$. In obese patients with T2DM weight loss improves glycaemic control and can reduce antidiabetic drug administration, especially in the early phase of the disease [11]. Some older antidiabetic drugs, such as sulfonylureas, glinides, thiazolidinediones and insulin result in weight gain [12]. Conversely, glucagon-like peptide receptors agonists (GLP-1ra), as well as sodium-glucose co- transporter 2 inhibitors (SGLT2i), result in average weight loss. Although few clinical trials reported a small mean weight loss $(0.5-1 \mathrm{~kg})$, metformin and dipeptidyl peptidase- 4 inhibitors (DPP-IVi) are considered weight neutral medications. In any case, drugs for the treatment of obesity should only be offered in a multicomponent lifestyle programme [13].

\section{Pharmacological therapy}

Glucagon-like peptide 1 receptor agonists: GLP-1ra is produced by L cells from small and large intestine, as well as from neurons within the nucleus of the solitary tract in the brainstem. GLP-1ra slow emptying gastric and cause an anorexic action bringing weight loss. The increased satiety is brought by both inhibition of emptying gastric and activation of receptor in the central nervous system. These compounds also enhance insulin secretion in a glucose-dependent way, avoiding, therefore, the risk of hypoglycaemia. The DPP-IV enzyme rapidly degrades human GLP-1 and its half-life is about 1-2 minutes. The development of subcutaneous products with longer half-life improved not only glycaemic control in T2DM, but may also induce significant weight loss. Intra-class differences may exist in relation to their effect of weight loss. It is possible to divide GLP-1ra in two categories: long- acting (semaglutide, dulaglutide, liraglutide, albiglutide, exenatide LAR) and short-acting (lixisetide, exenatide). Liraglutide and dulaglutide, approved by the US Food and Drug Administration (FDA) for T2DM, carry a weight loss of 1.3 to $8.65 \mathrm{~kg}, 2.3$ to $3 \mathrm{~kg}$, respectively when compared to baseline in a diabetic population with 6-12 months follow-up [14,15]. Currently, FDA, and also the European Medicines Agency (EMA), approved liraglutide 3mg/ day for treatment of obesity, because it produced an $-8.4 \mathrm{~kg}$ weight reduction also in a non-diabetic population [16]. Common side effects are nausea and vomiting which may occur at the beginning of the treatment. Semaglutide ( $1 \mathrm{mg} /$ weekly) is nowadays approved for T2DM therapy and is similar to liraglutide, displaying, however, a longer half time and inducing a significant weight loss from baseline $(-8.5 \mathrm{~kg})$ as liraglutide [17]. Semaglutide is the only oral GLP-1 agonist and at the dose of $14 \mathrm{mg}$ once daily produces a greater weight loss than the maximum dose of liraglutide approved for the treatment of type 2 diabetes. Semaglutide at $1.0 \mathrm{mg}$ once weekly seems more efficient in weight loss than dulaglutide $1.5 \mathrm{mg}$ once weekly. In fact, they caused a weight loss of $-6.5 \mathrm{~kg}$ and $-3.0 \mathrm{~kg}$ from baseline, respectively [18]. Exenatide carries lower weight loss activity by $-4.5 \mathrm{~kg}$, as underlined by the DURATION 1 Trial [19], compared with albiglutide $(-1.7 \mathrm{~kg}$ from baseline), as described by the study of Rosenstock et al. [20]. Also lixisenatide once daily caused weight loss of $-2.96 \mathrm{~kg}$ from baseline [21].

Sodium-glucose cotransporter 2 inhibitors: SGLT2i, inhibiting renal glucose transporter, induce a loss of average $50-80 \mathrm{~g}$ of glucose in urine per day and bring an osmotic diuresis associated with a loss of the body water. The most used SGLT2i are canaglifozin, dapaglifozin, empaglifozin. However, the weight loss observed is less than expected, probably due to a compensatory increase in food intake in patients treated with SGLT2i [22]. Indeed, several meta-analysis reported an average weight loss, observed in patients treated with SGLT2i, ranging from 0.591 to $2.1 \mathrm{~kg}$. The weight loss seems to occur rapidly in the early weeks of treatment, and then becomes more gradual. In the paper of Bolider et al., dapaglifozin causes a weight loss of $-2.42 \mathrm{~kg}$ vs. placebo at 102 weeks follow-up [23]. Long-term studies, verified by X-ray absorptiometry, support that dapaglifozin induces loss of fat mass $(1.34 \mathrm{~kg})[23]$. 
Other studies, including patients treated also with ipraglifozin underline that there is a loss of visceral fat induced by SGLT2i of about $-1.7 \mathrm{~kg}$ from baseline [24]. The EMPAREG-OUTCAME trial with empaglifozin, at 10 or $25 \mathrm{mg}$ once daily, showed an average weight loss of about 2 or $3 \mathrm{~kg}$, respectively vs. $1 \mathrm{~kg}$ in the placebo arm [25]. In the CANVAS study, canaglifozin displayed an average weight loss of about $-1.6 \mathrm{~kg}$ [26]. Finally, the DURATION 8 trial underlines how the combination metformin plus dapaglifozin once daily, combined with exenatide once weekly, resulted a $2 \%$ reduction of glycated haemoglobin and a weight loss of $3.5 \mathrm{~kg}$ in 28 weeks of treatment. These beneficial changes are more sustained in combined therapy than in monotherapy [27]. Also the study SUSTAIN 9 [28], a double-blind trial, parallel-group, noticed that GLP-1ra plus SGLT2i combination is more powerful in the reduction of body weight and of glycated haemoglobin compared to the add one to metformin and sulphonylurea.

Metformin: Metformin is used as a first-line anti-diabetic drug. Metformin reduces hepatic gluconeogenesis, decreases intestinal absorption of glucose and improves peripheral glucose uptake. Since patients with obesity generally develop insulin resistance, metformin can correct this alteration, reducing circulating insulin and promoting weight loss. Indeed, initial study at time of FDA approval, evidenced a moderate effect of metformin on weight control [29], whereas the UKPDS study reported a neutral effect on weight [30]. Although the statistical significance was not reached, some meta-analyses have shown a reduction of weight in patients treated with metformin [31,32]. The DPP (Diabetes and Prevention Program) identified a weight loss (participants had reduced body weight and waist circumference compared with placebo) with metformin, closely related with therapeutic adherence [33]. Given to the paucity of evidence, the FDA did not approved metformin as weight loss treatment. However, the ADA guidelines suggest metformin therapy for prevention of T2DM in subjects with "prediabetes", especially for those displaying a BMI $\geq 35 \mathrm{~kg} / \mathrm{m}^{2}$ [34].

\section{Discussion and Conclusion}

Most T2DM cases are related to over-weight or obesity, therefore, it is really important to promote weight loss with nutritional advices, as well as drugs. Significant interventions on patient's lifestyle are crucial to treat both diabetes and obesity that can be seen as the two faces of the same disease, the metabolic syndrome. Nowadays some drugs can help clinicians to treat both diseases; among these, the most effective are GLP-1ra, in terms of both reduction of glycated haemoglobin and weight loss, due to their mechanism of action. In particular, the most effective molecules are liraglutide and semaglutide. The combination therapy GLP-1ra and SGLT2i, considering their specific mechanistic synergy, can result in further reduction of glycated haemoglobin and weight, without any hypoglycaemic risks, as shown in the meta-analysis by Castellana et al. [35]. GLP-1ra and SGLT2i can bring cardiovascular advantage, strongly needed in this population with high cardiovascular risk.
Metformin is always first-line therapy in T2DM for its tolerability, no hypoglycaemic risks and considerable action reducing glycated haemoglobin. Thanks to their different mechanism of action, metformin in association with GLP-1ra and/or SGLT2i probably represent the best choice for our patients with T2DM and obesity.

\section{References}

1. Haslam D (2010) Obesity and diabetes: the links and common approaches. Primary Care Diabetes 4(2):105-112.

2. Riobo Servan $P$ (2013) Obesity and diabetes. Nutricion Hospitalaria 28 (Suppl 5): 138-143.

3. Skyler JS, Bakris GL, Bonifacio E, Darsow T, Eckel RH, et al. (2017) Differentiation of diabetes by pathophysiology, natural history, and prognosis. Diabetes 66(2): 241-255.

4. Defronzo RA (2009) From the triumvirate to the ominous octet: a new paradigm for the treatment of type 2 diabetes mellitus. Diabetes 58(4): 773-795.

5. Kashyap SR, Defronzo RA (2007) The insulin resistance syndrome: physiological considerations. Diabetes \& Vascular Disease Research 4(1): 13-19.

6. Huang PL (2009) A comprehensive definition for metabolic syndrome. Disease Models \& Mechanisms 2(5-6): 231-237.

7. Forga L, Petrina E, Barberia JJ (2002) Complications of obesity. Annals of the Health System of Navarra 25 (Suppl 1):117-126.

8. American Diabetes Association (2018) 7. Obesity management for the treatment of type 2 diabetes: standards of medical care in diabetes-2018. Diabetes Care 41(Suppl 1): S65-S72.

9. Donnelly JE, Blair SN, Jakicic JM, Manore MM, Rankin JW, et al. (2009) American College of Sports Medicine Position Stand. appropriate physical activity intervention strategies for weight loss and prevention of weight regain for adults. Medicine and Science in Sports and Exercise 41(2): 459-471.

10. Lee CD, Blair SN, Jackson AS (1999) Cardiorespiratory fitness, body composition, and all-cause and cardiovascular disease mortality in men. The American Journal of Clinical Nutrition 69(3): 373-380.

11. Pastors JG, Warshaw H, Daly A, Franz M, Kulkarni K (2002) The evidence for the effectiveness of medical nutrition therapy in diabetes management. Diabetes care 25(3): 608-613.

12. Palmer SC, Mavridis D, Nicolucci A, Johnson DW, Tonelli M, et al. (2016) Comparison of clinical outcomes and adverse events associated with glucose-lowering drugs in patients with type 2 diabetes: A metaanalysis. JAMA 316(3): 313-324.

13. Wilding JPH (2018) Medication use for the treatment of diabetes in obese individuals. Diabetologia 61(2): 265-272.

14. Ostawal A, Mocevic E, Kragh N, Xu W (2016) Clinical effectiveness of liraglutide in type 2 diabetes treatment in the real-world setting: A systematic literature review. Diabetes Therapy: 7(3): 411-438.

15. Jendle J, Grunberger G, Blevins T, Giorgino F, Hietpas RT, et al. (2016) Efficacy and safety of dulaglutide in the treatment of type 2 diabetes: A comprehensive review of the dulaglutide clinical data focusing on the AWARD phase 3 clinical trial program. Diabetes/Metabolism Research and Reviews 32(8): 776-790.

16. Pi Sunyer X, Astrup A, Fujioka K, Greenway F, Halpern A, et al. (2015) A randomized, controlled trial of $3.0 \mathrm{mg}$ of liraglutide in weight management. N Engl J Med 373(1): 11-22.

17. O Neil PM, Birkenfeld AL, McGowan B, Mosenzon O, Pedersen SD, et al (2018) Efficacy and safety of semaglutide compared with liraglutide and placebo for weight loss in patients with obesity: A randomised, doubleblind, placebo and active controlled, dose-ranging, phase 2 trial. Lancet 392(10148): 637-649. 
18. Pratley RE, Aroda VR, Lingvay I, Ludemann J, Andreassen C, et al. (2018) Semaglutide versus dulaglutide once weekly in patients with type 2 diabetes (SUSTAIN 7): A randomised, open-label, phase $3 \mathrm{~b}$ trial. Lancet Diabetes \& Endocrinology 6(4): 275-286.

19. Buse JB, Drucker DJ, Taylor KL, Kim T, Walsh B, et al. (2010) DURATION-1: exenatide once weekly produces sustained glycemic control and weight loss over 52 weeks. Diabetes Care 33(6): 1255-1261.

20. Rosenstock J, Reusch J, Bush M, Yang F, Stewart M, et al. (2009) Potential of albiglutide, a long- acting GLP-1 receptor agonist, in type 2 diabetes: A randomized controlled trial exploring weekly, biweekly, and monthly dosing. Diabetes Care 32(10): 1880-1886.

21. Trujillo JM, Goldman J (2017) Lixisenatide, a once-daily prandial glucagon-like peptide-1 receptor agonist for the treatment of adults with type 2 diabetes. Pharmacotherapy 37(8): 927-943.

22. Ferrannini G, Hach T, Crowe S, Sanghvi A, Hall KD, et al. (2015) Energy balance after sodium- glucose cotransporter 2 inhibition. Diabetes Care 38(9): 1730-1735.

23. Bolinder J, Ljunggren O, Johansson L, Wilding J, Langkilde AM, et al (2014) Dapagliflozin maintains glycaemic control while reducing weight and body fat mass over 2 years in patients with type 2 diabetes mellitus inadequately controlled on metformin. Diabetes, Obesity \& Metabolism 16(2): 159-169.

24. Yamamoto C, Miyoshi H, Ono K, Sugawara H, Kameda R, et al. (2016) Ipragliflozin effectively reduced visceral fat in Japanese patients with type 2 diabetes under adequate diet therapy. Endocrine Journal 63(6): 589-596.

25. Zinman B, Wanner C, Lachin JM, Fitchett D, Bluhmki E, et al. (2015) Empagliflozin, cardiovascular outcomes, and mortality in type 2 diabetes. N Engl J Med 373(22): 2117-2128.

26. Neal B, Perkovic V, Mahaffey KW, de Zeeuw D, Fulcher G, et al. (2017) Canagliflozin and cardiovascular and renal events in type 2 diabetes. $\mathrm{N}$ Engl J Med 377(7): 644-657.
27. Frías JP, Guja C, Hardy E, Ahmed A, Dong F, et al. (2016) Exenatide once weekly plus dapagliflozin once daily versus exenatide or dapagliflozin alone in patients with type 2 diabetes inadequately controlled with metformin monotherapy (DURATION-8): A 28 week, multicentre, double-blind, phase 3 , randomised controlled trial. Lancet Diabetes Endocrinol 4(12): 1004-1016.

28. Zinman B, Bhosekar V, Busch R, Holst I, Ludvik B, et al. (2019) Semaglutide once weekly as add-on to SGLT-2 inhibitor therapy in type 2 diabetes (SUSTAIN 9): A randomised, placebo-controlled trial. The Lancet Diabetes \& Endocrinology 7(5): 356-367.

29. Stumvoll M, Nurjhan N, Perriello G, Dailey G, Gerich JE (1995) Metabolic effects of metformin in non-insulin-dependent diabetes mellitus. N Engl J Med 333(9): 550-554.

30. Holman RR, Paul SK, Bethel MA, Matthews DR, Neil HA (2008) 10-year follow-up of intensive glucose control in type 2 diabetes. $N$ Engl J Med 359(15): 1577-1589.

31. Kahn SE, Haffner SM, Heise MA, Herman WH, Holman RR, et al. (2006) Glycemic durability of rosiglitazone, metformin, or glyburide monotherapy. N Engl J Med 355(23): 2427-2443.

32. Saenz A, Fernandez Esteban I, Mataix A, Ausejo M, Roque M, et al. (2005) Metformin monotherapy for type 2 diabetes mellitus. The Cochrane Database of Systematic Reviews (3):CD002966.

33. Diabetes Prevention Program Research Group (2012) Long-term safety, tolerability, and weight loss associated with metformin in the diabetes prevention program outcomes study. Diabetes Care 35(4): 731-737.

34. American Diabetes Association (2019) 3. Prevention or delay of type 2 diabetes: standards of medical care in diabetes-2019. Diabetes Care 42 (Suppl 1): S29-S33.

35. Castellana M, Cignarelli A, Brescia F, Perrini S, Natalicchio A, et al. (2019) Efficacy and safety of GLP- 1 receptor agonists as add-on to SGLT2 inhibitors in type 2 diabetes mellitus: A meta-analysis. Scientific Reports 9(1): 19351. 